\title{
LOSSY COMPRESSION SCHEMES BASED ON TRANSFORMS-A LITERATURE REVIEW ON MEDICAL IMAGES
}

\author{
Sherin Jabbar ${ }^{1}$ and Shaiju Panchikkil ${ }^{2}$ \\ Department of Computer Engineering, MES Engineering College, Kuttippuram, Kerala
}

\begin{abstract}
In recent years, advances in information technology and telecommunications have played a crucial role as catalysts for significant developments in the sector of healthcare. These technological advances have played a very strong role in the field of medical imaging. The number of digital medical images has increased rapidly on the Internet. The necessity of fast and secure diagnosis is vital in the medical world. The purpose of medical image compression is express medical images with less data so that it can be efficiently stored and transmitted. Diagnosis is effective only when compression techniques preserve all the relevant and important image information needed. There are basically two types of image compression: lossless and lossy. Lossless coding does not permit high compression ratios where as lossy achieve high compression ratio. Among the existing lossy compression schemes, transform coding is one of the most effective strategies. This paper summarizes the different compression methods based on transforms like Discrete Cosine Transform(DCT), Discrete Wavelet Transform(DWT), Hybrid DCT-DWT and Contourlet transform. And it has been analyzed that Contourlet transform have superior overall performance over other transforms in terms of PSNR.
\end{abstract}

\section{KEYWORDS}

Medical Image Compression, Wavelet Transform, Discrete Cosine Transform, Contourlet Transform

\section{INTRODUCTION}

With the development in Internet and multimedia technologies, the amount of information that is handled by computers has grown very fast. This information requires large amount of storage space and transmission bandwidth. One of the possible solutions to this problem is to compress the information so that the storage space and transmission time can be reduced. Major part of this information that has to store and transmit includes images which have larger size. So image compression will solve these issues regarding storage and transmission. Recent years, advances in information technology and telecommunications have played a crucial role as catalysts for significant developments in the sector of healthcare. These technological advances have played a very strong role in the field of medical imaging. The number of digital medical images has increased rapidly on the Internet. The necessity of fast and secure diagnosis is vital in the medical world. Due to rapid increase in medical data produced by hospitals and because of the high cost of providing a large transmission bandwidth and huge amount of storage space, compression of images is becoming increasingly important. The purpose of medical image compression is to express images with less data to save storage space and transmission time, based on the premise that true information in the original image will be preserved. Especially in medical imaging application diagnosis is effective only when the compression technique preserve all relevant and important information needed [1]. Two ways of classifying compression techniques are mentioned here: 
(a) Lossless vs. Lossy compression: In lossless compression schemes, the reconstructed image, after compression, is numerically identical to the original image. However lossless compression can only a achieve a modest amount of compression. An image reconstructed following lossy compression contains degradation relative to the original. Often this is because the compression scheme completely discards redundant information. However, lossy schemes are capable of achieving much higher compression. Under normal viewing conditions, no visible loss is perceived (visually lossless).

(b) Predictive vs. Transform coding: In predictive coding, information already sent or available is used to predict future values, and the difference is coded. Since this is done in the image or spatial domain, it is relatively simple to implement and is readily adapted to local image characteristics. Differential Pulse Code Modulation (DPCM) is one particular example of predictive coding. Transform coding, on the other hand, first transforms the image from its spatial domain representation to a different type of representation using some well-known transform and then codes the transformed values (coefficients). This method provides greater data compression compared to predictive methods, although at the expense of greater computation.

\subsection{Lossy Image Compression}

Compression methods used in medical applications are most of the time lossless methods in order to preserve the data integrity and to facilitate a true diagnosis. However, lossless coding does not permit high compression ratios. Thus, most of applications such as telemedicine and fast searching and browsing of medical volumetric data suffer from this limitation [3]. However, lossy coding can achieve higher compression. Among the existing compression schemes, transform coding is one of the most effective strategies.

A typical lossy compression system mainly consists of three major steps: First step is the transformation which is the step that gives rise to higher compression. The second process is quantization which is the key issue for lossy methods and it is the difference between lossless and lossy methods. Quantization reduces the symbols used to represent the image. Last part of lossy compression process is entropy encoding. Quantized symbols are encoded using different entropy coding algorithms, like Huffman encoding.

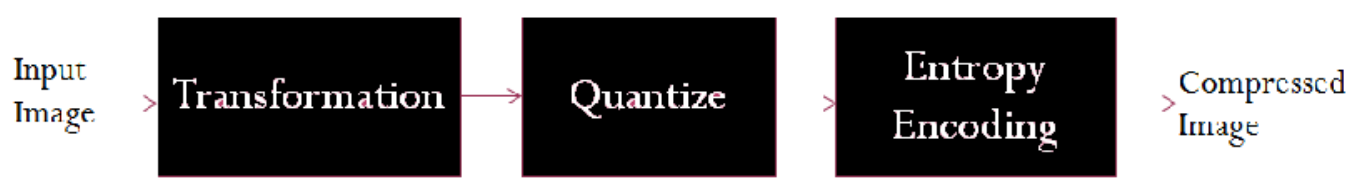

In digital images the spatial frequencies are important as the low-frequency components correspond to important image features and the high-frequency ones to image details. High frequencies are a less important part of the images and can be quantized more heavily than low frequency coefficients to achieve low-bit rates. A linear transformation matrix [W], whose transpose $[\mathrm{w}]^{\mathrm{T}}$ will rotate the data matrix $\mathrm{X}$ to produce a diagonal covariance matrix for the transformed variable $\mathrm{Y}$ where $\mathrm{X}=\left[\mathrm{x}_{1}, \mathrm{x}_{2}, \mathrm{x}_{3}, \mathrm{x}_{4} \ldots . \mathrm{x}_{\mathrm{N}}\right]^{\mathrm{T}}$ is a vector having $\mathrm{N}$ pixel or data points. Then, $Y=[W]^{T} X$. Each column vector $w_{i}$ of $W$ is a basis vector of new space. So alternatively each element $y_{i}$ of $Y$ calculated as $w_{i}^{T} X$. The inverse transformation is calculated as $X=[W] Y$

There are various transforms like discrete cosine transform, discrete wavelet transform, Contourlet transform that are used for effective compression. In this paper, a review on compression techniques using different transform like discrete cosine transform (DCT), discrete wavelet transform (DWT), and Contourlets transform are reviewed and their effectiveness on medical image compression have been studied. The remainder of the paper proceeds as follows: 
International Journal of Advanced Information Technology (IJAIT) Vol. 2, No.6, December 2012

Section 2 covers Literature survey and observation and Analysis. Section 3 presents the future scope of work and Conclusion.

\section{REVIEW}

Compression using Discrete Cosine Transform (DCT) [2] divides up the image into 8 by 8 pixel blocks and then calculates the discrete cosine transform (DCT) of each block. A quantizer rounds of the DCT coefficients according to the quantization matrix. This step produces the "lossy" nature, but allows for large compression ratios. This compression technique uses a variable length code on these coefficients, and then writes the compressed data stream to an output file. For decompression, it recovers the quantized DCT coefficients from the compressed data stream, takes the inverse transforms and displays the image. Figure 1 shows this process. DCT is given by the equation 2.1:

$$
D(u, v)=\sqrt{\frac{2}{N}} \sqrt{\frac{2}{M}} C(u) C(v) \sum_{i=1}^{N} \sum_{j=1}^{M} P(x, y) \cos \left[\frac{(2 x+1) \Pi u}{2 N}\right] \cos \left[\frac{(2 y+1) \Pi v}{2 M}\right]
$$

Where $c(i)=\left\{\begin{array}{c}\frac{1}{\sqrt{2}}, i=0 \\ 1, i>0\end{array}\right.$

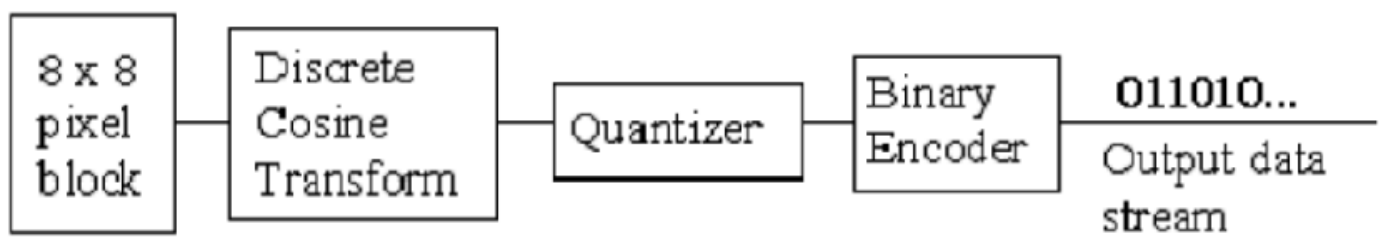

Figure 1: Compression using DCT

Elham Shahhoseini [3] presents a new lossy technique based on wavelet transform for compression of breast ultrasound images. The experiments are performed on 16 different wavelet functions and the quality of reconstructed images is evaluated by using Compression Ratio (CR), Normalized Mean Square Error (NMSE), Normalized Absolute Error (NAE), and Peak Signal to Noise Ratio (PSNR) criterion. Two dimensional wavelet transform is usually performed by applying a separable filter bank to the image. Typically, a low pass filter and a high pass filter are used ( $h$ and g, respectively). A group of transforms coefficients resulting from the same sequence of low pass and high pass filtering operations, both horizontally and vertically are called subbands. Applying the one dimensional transform in each row, produce two subbands in each row ( $\mathrm{L}$ and $\mathrm{H}$ subbands). Then applying a one dimensional DWT column-wise on these $\mathrm{L}$ and $\mathrm{H}$ subbands (intermediate result), produce four subbands LL, LH, HL, and HH. LL is a coarser version of the original input signal called approximation image. LH, HL, and HH are the high frequency subbands containing the detail information (details images). The number of decompositions performed on original image to obtain subbands is called subband decomposition level. The method used to perform lossy image compression via wavelet thresholding consists of the following processes: The images are initially transformed by wavelet transform at one level. Then the threshold value, Thr, based on the transform coefficients is defined by (2.3). 
International Journal of Advanced Information Technology (IJAIT) Vol. 2, No.6, December 2012

$$
\begin{aligned}
& \text { Thr }=C(r) \\
& C_{\text {new }}=c_{i} \mid \forall_{i}<T h r, C(i)=0
\end{aligned}
$$

where $\mathrm{n}$ is the number of detail coefficients, $\mathrm{C}$ is the wavelet coefficient vector and $\mathrm{r}$ is the remaining rate in percent. Then the renewed wavelet coefficients $\mathrm{C}_{\text {new }}$ is as described in (2.4). In this work, the compression process is done at $10 \%$ and $20 \%$ remaining rate.

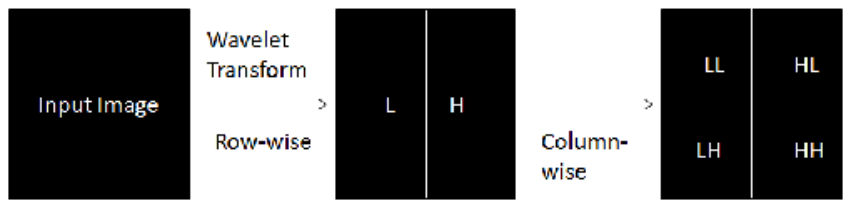

Figure 2: Decomposition of wavelet transform

Aree Ali Mohammed et al [4] present a scheme for medical image compression based on hybrid compression technique (DWT and DCT). The goal is to achieve higher compression rates by applying different compression thresholds for the wavelet coefficients of each DWT band (LL and $\mathrm{HH}$ ) while DCT transform is applied on ( $\mathrm{HL}$ and $\mathrm{LH}$ ) bands with preserving the quality of reconstructed medical image. The retained coefficients are quantized by using adaptive quantization according to the type of transformation. Finally the entropy coding (variable shift coding) is used to encode the quantization indices. A novel algorithm for medical image compression is developed using 917 Tap wavelet filter, DCT transform and optimized entropybased coding technique [9]. Step1: color space conversion. In order to compress bandwidth, $\mathrm{C}_{\mathrm{b}}$ and $\mathrm{C}_{\mathrm{r}}$ are sampled at a lower rate than $\mathrm{Y}$, which is technically known as "chroma subsampling." This means that some color information in the image is being discarded, but not brightness (luma) information. Step2: Forward Discrete Wavelet Transform (FDWT) is applied to the image. After applying FDWT on the medical images data, one can obtain different level of bands. LL and HH bands coefficients are directly sent to the adaptive quantizer according to the nature of bands. The remaning bands (HL and $\mathrm{LH}$ ) coefficients are subjected to DCT transformation. Each of HL and LH bands are divided into 8X8 blocks and converted to frequency domain using 2D FDCT as in equation 2.1. Both DCT(HL,LH) and DWT(LL,HH) band coefficients are then quantized. The LL, HH coefficients must be quantized using adaptive quantization. The luminance component $\mathrm{Y}$ requires the small step of quantization while $\mathrm{Cb}$ and $\mathrm{Cr}$ need a large step. After this step, a large sequence of zeros is obtained especially in HH part of the image. Step4: The forward differential pulse code modulation is applied on the quantized (LL band) wavelet coefficients and quantized DC coefficients of DCT transform. And then all the coefficients must be converted into positive values by mapping to positive technique. Step5: The proposed coding scheme is a variable shift coding technique which gives a few bits to the short codeword and many bits to the long codeword. The reconstructed image is obtained by applying the inverse steps of coding process.

SeyyedHadi Hashemi-Berenjabad et al[5] present a new Contourlet based lossy image compression method for medical ultrasound images. In this algorithm Contourlet transform for image decomposition is used. In [5] Contourlet transform is used to decompose the image into coefficients. After decomposition, a threshold is chosen from Contourlet coefficients. Then, a thresholding process is applied on the coefficients and routine quantization process is performed. In this algorithm choosing the threshold plays a significant role. So, in order to minimize the important information loss the most frequently occurring Contourlet coefficient of the image is selected as the threshold. After thresholding the coefficients is quantized. Huffman coding is applied on quantized coefficients and the coded bit-stream is generated. This threshold based process reduces the number of coefficients necessary to reconstruct the image. Scalar quantization 
International Journal of Advanced Information Technology (IJAIT) Vol. 2, No.6, December 2012

is selected due to its simplicity and performance. The coded bit-stream includes coefficients and Contourlet filter information. The Contourlet transform is a directional transform, capable of capturing contours and fine details in images. The Contourlet expansion is composed of basis function oriented at various directions in multiple scales, with flexible aspect ratios. Using this rich set of basic functions, the Contourlet transform effectively capture the smooth contours that are dominant feature in images [8]. The Contourlet uses a double filter-bank structure, namely pyramidal directional filter banks (PDFB) which is composed of Laplacian Pyramid (LP) and Directional Filter Banks (DFB). LP implements multiresolution decomposition to generate multiscale representation of the image. PDFB uses DFB to process subband images from LP and reveal directional details in each scale level.

\subsection{Observation and Analysis}

Evaluation of the diagnostic quality of compressed medical image still re-mains an important issue. However, two measurement methods have dominated the assessment of medical image quality, which are computable objective distortion measure and subjective quality as measured by psychophysical tests [4]. The objective quality of a reconstructed image could be measured by the Peak Signal to Noise Ratio (PSNR) between the original and reconstructed images. Compression Ratio (CR) is equal to the size of the compressed image divided by the size of the original image.

\subsubsection{Peak Signal to Noise Ratio}

The objective performance is measured by peak signal-to-noise-ratio (PSNR) of the reconstructed image. PSNR measured in decibels $(\mathrm{dB})$ is given by:

$$
P S N R=10 \log 10 \frac{(M A X)^{2}}{M S E}
$$

Where MAX is the maximum gray level value of the image and MSE is the mean square error between the original image and reconstructed image which is defined by

$$
M S E=\frac{1}{M N} \sum_{m=o}^{M-1} \sum_{n=o}^{N-1}\left|x(m, n)-x_{q}(m, n)\right|^{2}
$$

Where $\mathrm{x}(\mathrm{m}, \mathrm{n})$ is the original image and $\mathrm{x}_{\mathrm{q}}(\mathrm{m}, \mathrm{n})$ is the reconstructed image and $\mathrm{M}^{*} \mathrm{~N}$ is the size of original image.

For a lower compression ratio, DCT based image compression yielded higher quality image than Wavelet. While the DCT-based image JPEG coders perform very well at moderate bit rates, at higher compression ratios, image quality de-grades because of the artifacts resulting from the block-based DCT scheme. For CT scan image DCT based compression method outperforms the Peak Signal to Noise Ratio (PSNR) and degree of compression than wavelet compression method.

Wavelet-based coding is more robust under transmission and decoding errors, and also facilitates progressive transmission of images. In addition, they are better matched to the Human Visual System characteristics [9]. Because of their inherent multi resolution nature, wavelet coding schemes are especially suitable for applications where scalability and tolerable degradation are important $[10,11]$. Good applications for wavelet compression are large images, images with low-contrast edges e.g., medical images. It offers numerous benefits over current compression methods, including the ability to do both lossless and lossy compression, the ability to obtain 
International Journal of Advanced Information Technology (IJAIT) Vol. 2, No.6, December 2012

higher image quality and higher compression ratios, and the ability to view the same file at multiple resolutions. Wavelets are good at isolating the discontinuities at edge points, but will not see the smoothness along the contours [5].

The hybrid using DCT-DWT compression technique is tested against different medical images using different values of compression factors (i.e. DWT and DCT quantization factors). As the quantization factors increase the Compression ratio increase and the quality measurement (PSNR) decrease [4].

Contourlets not only possess the main features of wavelets (i.e. multi-scale and time-frequency localization), but also offer a high degree of directionality and anisotropy. Similar to wavelet, Contourlet can decompose the image into different scales. But unlike the wavelet which can only decompose each scale into two directions, Contourlet can decompose each scale into any arbitrarily power of two's number of directions and different scales can be decomposed into different number of directions [5]. Contourlet transform produces more data related to original data, which is not the case for wavelet transform, the entropy of subbands in Contourlet transform is much less than that of wavelet transform. Besides, the Contourlet preserves better the edges than wavelet causing better PSNR. So, these two facts cause that the overall performance of Contourlet transform is better for the compression of CT images [9]. However, at lower compression ratios, effect of producing more data in Contourlet transform is dominant which causes that wavelet and Contourlet transform produce nearly the same results. The results reveal the superior overall performance of Contourlet against wavelet transform at higher compression ratios. However at lower compression ratios wavelet transform is still suitable approach. Contourlets offer a much richer set of directions and shapes, and thus they are more effective in capturing smooth contours and geometric structures in images [8]. It has been observed that proposed method using Contourlets has acceptable performance and good performance over common compression methods.

\section{Conclusions}

The observation from literature survey revealed superior performance of Contourlet transform over other transforms in terms of PSNR and compression ratio. There is a superior overall performance of Contourlet against wavelet transform at higher compression ratios. However at lower compression ratios wavelet transform is still suitable a approach [7]. Contourlets offer a much richer set of directions and shapes, and thus they are more effective in capturing smooth contours and geometric structures in images especially in medical images. Compression of images using Contourlet transform can be extended to real time application for video compression in medical images.

\section{ACKNOWLEDGEMENTS}

I wish to express my deep sense of gratitude to Our Prinicipal, Dr. V.H. Abdul Salam, Our Head of Department Dr.P.P Abdul Haleem and Our Project Co-ordinator Mr. Lijo V. P for their support with their immense knowledge. Words are inadequate in offering my thanks to Mr. Shaiju Panchikkil, Assistant Professor, who had been a source of inspiration for his timely guidance and encouragement. All glory and honor to Almighty God. 
International Journal of Advanced Information Technology (IJAIT) Vol. 2, No.6, December 2012

\section{REFERENCES}

[1] M. R. B. Puja Bharti, Dr. Savith Gupta, "Comparative analysis of image compression techniques: a case study on medical images," in International Conference on Advances in Recent Technologies in Communication and Computing, 2009. ARTCom '09. IEEE, 2009, pp. 820-822.

[2] N. Thota and S. Devireddy, "Image compression using discrete cosine trans-form," Georgian Electronic Scientific Journal: Computer Science and Telecommunications,vol. 17, no. 3,pp.35-43, 2008.

[3] E. Shahhoseini, N. Nejad, H. Behnam, and A. Shahhoseini, "A new approach to compression of medical ultrasound images using wavelet transform," in 2010 Third International Conference on Advances in Circuits, Electronics and Micro-Electronics (CENICS),. IEEE, 2010, pp. 40-44.

[4] A. Mohammed and J. Hussein, "Hybrid transform coding scheme for medical image application," in 2010 IEEE International Symposium on Signal Processing and Information Technology (ISSPIT). IEEE, 2010, pp. 237-240.

[5] S. Hashemi-Berenjabad, A. Mahloojifar, and A. Akhavan, "Threshold based lossy compression of medical ultrasound images using contourlet transform," in 2011 18th Iranian Conference of Biomedical Engineering (ICBME). IEEE,Dec 2011, pp. 191-194.

[6] A. Graps, "An introduction to wavelets," Computational Science \& Engineer-ing, IEEE, vol. 2, no. 2, pp. 50-61, 1995.

[7] J. Walker and T. Nguyen, "Wavelet-based image compression," The Trans-form and Data Compression Handbook Ed. KR Rao et al. Boca Raton, CRC Press LLC, 2001.

[8] M. Do and M. Vetterli, "The contourlet transform: an efficient directional multiresolution image representation, "Image Processing, IEEE Transactions on, vol. 14, no. 12, pp. 2091-2106, 2005.

[9] N. Riazifar and M. Yazdi, "Effectiveness of contourlet vs wavelet transform on medical image compression: a comparative study," World Academy of Science, Engineering and Technology, vol. 49, pp. 837-842, May 2009.

\section{Authors}

Sherin Jabbar ${ }^{1}$, presently pursuing M-tech degree from MES Engineering College, Kuttippuram, Kerala under Calicut University. She is having total teaching experience of two years.

Shaiju Panchikkil 2, working as Asst. Professor, MES College of Engineering, Kuttippuram, Kerala. He did his ME in CSE from Paavai College of Engg., Namakkal, TN and B.Tech from MEA Engg. College, Perinthalmanna, Malappuram, Kerala. He has got an Experience of $3.5 y r s$ in the IT industry.

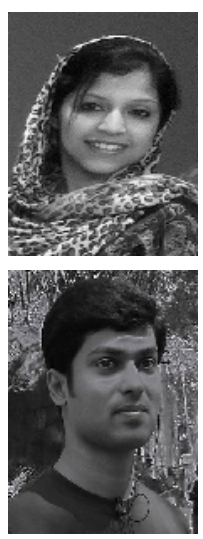

